\title{
mTORC2 modulates feedback regulation of p38 MAPK activity via DUSP10/MKP5 to confer differential responses to PP242 in glioblastoma
}

\author{
Angelica Benavides-Serrato ${ }^{1,4}$, Lauren Anderson ${ }^{4}$, Brent Holmes ${ }^{4}$, Cheri Cloninger ${ }^{4}$, \\ Nicholas Artinian", Tariq Bashir ${ }^{1,4}$ and Joseph Gera ${ }^{1,2,3,4}$ \\ ${ }^{1}$ Department of Medicine, David Geffen School of Medicine, University of California, Los Angeles, CA, USA \\ 2 Jonnson Comprehensive Cancer Center, University of California, Los Angeles, CA, USA \\ ${ }^{3}$ Molecular Biology Institute, University of California, Los Angeles, CA, USA \\ ${ }^{4}$ Division of Hematology-Oncology, Greater Los Angeles Veterans Affairs Healthcare System, Los Angeles, CA, USA \\ Correspondence to: Joseph Gera, email: jgera@mednet.ucla.edu \\ Keywords: p38 MAPK/DUSP/mTOR
}

Received: August 26, 2014

Accepted: October 22, 2014

Published: October 23, 2014

This is an open-access article distributed under the terms of the Creative Commons Attribution License, which permits unrestricted use, distribution, and reproduction in any medium, provided the original author and source are credited.

ABSTRACT

Dual-specificity phosphatases (DUSPs) dephosphorylate MAP kinases (MAPKs) resulting in their inactivation. Activation of MAPK signaling leads to enhanced DUSP expression, thus establishing feedback regulation of the MAPK pathway. The DUSPs are subject to regulation at the post-translational level via phosphorylation resulting in alterations of protein stability. Here we report that mTORC2 function leads to stabilization of the p38 MAPK phosphatase, DUSP10, thereby inhibiting p38 activity. We demonstrate that MTORC2 binds DUSP10 and phosphorylates DUSP10 on serine residues 224 and 230. These phosphorylation events block DUSP10 turnover resulting in inactivation of p38 signaling. We further show that insulin-stimulated PI3K/mTORC2 signaling regulates DUSP10 stability and p38 activity. Importantly, knockdown of DUSP10 or ectopic overexpression of nonphosphorylatable or phosphomimetic DUSP10 mutants was sufficient to confer differential mTOR kinase inhibitor responses to GBM cells in vitro and in murine xenografts. Finally, DUSP10 was shown to be overexpressed in a significant number of GBM patients. These data demonstrate the ability of the mTORC2 pathway to exert regulatory effects on the DUSP10/p38 feedback loop to control the cellular effects of mTOR kinase inhibitors in GBM and support the use of DUSP10 expression as a surrogate biomarker to predict responsiveness.

\section{INTRODUCTION}

The p38 mitogen-activated protein kinases (MAPKs) are responsive to several stress stimuli such as UV irradiation, heat shock and cytokine exposure. They are involved in the processes of cellular differentiation, autophagy and apoptosis [1]. Dual-specificity MAPK kinase 3 and SAPK/ERK kinase activate p38 by phosphorylating threonine 180 and tyrosine 182 [2]. Conversely, the regulation of $\mathrm{p} 38$ signaling is dependent on the activity of several p38 phosphatases such as DUSP1 and DUSP10 which dephosphorylated these residues
[3]. It is hypothesized that these DUSPs constitute a negative feedback loop which functions to establish a threshold of MAP kinase activation through preemptive dephosphorylation of MAP kinases [3, 4]. This function has been proposed for DUSP3 as well as with non-DUSP phosphatases such as PP2A [4].

The regulation of DUSP activity is tightly controlled post-translationally, as the half-lives of many of the DUSPs is only approximately $60 \mathrm{~min}$ [5]. This tight regulation is partially mediated by the phosphorylation of DUSPs by MAP kinases that block the turnover of DUSPs by proteasomes [3]. This regulation of DUSP stability, coupled with their relatively short protein half- 
life and high inducibility suggests that DUSPs are likely to function as an immediate-early-off-switch regulating MAP kinase signaling [3]. Whether other signaling cascades can affect DUSP expression post-translationally is not completely understood.

The mTOR kinase is a central regulator of cell growth and size [6]. Regulation of mTOR signaling plays a major role in cancer and also in the cellular responses to nutrients, mitogens and chemotherapeutic agents. mTOR is present in two functionally and structurally individual multiprotein complexes termed TOR complex 1 (TORC1) and TORC2 [7]. The mammalian TOR complex 1 (mTORC1) contains mTOR, mLST8 and Raptor and is rapamycin sensitive. mTORC2 consists of Rictor, mSIN1, mLST8, PRR5 and mTOR and is rapamycin insensitive. Recently developed mTOR kinase inhibitors effectively block the activities of both complexes, thereby more dramatically inhibiting protein synthesis, suppressing AKT activation and inducing $\mathrm{G}_{1}$ arrest or apoptosis in tumor cells as compared to rapamycin [8]. mTORC1 signaling results in the phosphorylation of its downstream effectors $\mathrm{p} 70 / \mathrm{S} 6 \mathrm{~K}$ and 4E-BP1 [9]. Activation of mTORC2 leads to phosphorylation of the AKT kinase at serine 473 resulting in AKT's full activation [10].

mTOR signaling plays a critical role in the cellular response to various stresses [11]. mTORC1 regulates growth and cap-dependent mRNA translation via effects on p70S6K and 4E-BP1 and inhibits autophagy [12]. Signaling inputs sensing the relative availability of amino acids have been demonstrated to enter the mTORC1 pathway from the lysososome via the Rag GTPases [13]. Muscle contraction or fluid and sheer stresses induce p70/S6K activity, while heat shock, reactive oxygen intermediates, osmotic stresses and DNA damage generally decrease $\mathrm{p} 70 / \mathrm{S} 6 \mathrm{~K}$ function [14]. The mechanisms by which these latter stimuli signal to the mTORCs are not well understood.

In this report we demonstrate that Rictor and DUSP10 interact in yeast and mammalian cells and that DUSP10 is a substrate for mTORC2. We identified the phosphosites on DUSP10 and demonstrate that phosphorylation of these residues alters DUSP10 protein stability by blocking its degradation. We further demonstrate that stimulation of PI3K signaling, leading to mTORC2 activation, stabilizes DUSP10 and results in suppression of p38 MAP kinase activity supporting the ability of the mTOR pathway to regulate DUSP10 expression post-translationally. Finally, we show that enforced expression of either an mTORC2 nonphosphorylatable or phosphomimetic DUSP10 phosphosite mutant is sufficient to alter cellular responsiveness to mTOR kinase inhibitors.

\section{RESULTS}

\section{DUSP10 interacts with the Rictor component of mTORC2}

In a previous large-scale yeast two-hybrid screen for interactors of the mTORC2 regulatory subunit Rictor, we identified Hsp70 as a Rictor interacting protein which was demonstrated to be required for supracomplex assembly and activity under hyperthermic conditions [17]. To extend our screening efforts, we prepared cDNA libraries from cell lines which overexpressed Rictor and were dependent on mTORC2 signaling for growth in an effort to capture additional Rictor interactors which may have not been represented well in the libraries utilized in our previous study. Utilizing these cDNA libraries we identified most of the known Rictor interacting proteins as well as novel binding proteins. A majority of the total recovered clones identified the major cytoplasmic phosphatase for p38 MAPK, DUSP10 (MKP5) as a Rictor binding partner. Several other genetic interactors were identified and are listed in Table 1. To determine which regions were involved in the binding of Rictor to DUSP10 we generated a set of deletion mutants shown in figure 1A. Rictor or DUSP10 were fused to either the Gal4-activation domain (AD) or Gal4-DNA binding domain (DBD) and these constructs were introduced into the yeast two hybrid strain AH109, plated on selective media to assess growth and the relative strength of the interactions were determined by liquid $\beta$-galactosidase assays. As shown, a region close to the N-terminus of Rictor (a.a. 50-101) was required for binding with the full-length DUSP10AD fusion. A region of DUSP10, N-terminal to the MAP kinase binding domain (a.a. 101-146), was required for substantial reporter activity with full-length Rictor. Fusions of only the interacting domains within Rictor and DUSP10 to the Gal4-DBD or AD, respectively, were sufficient to mediate a robust interaction and led to significant growth and high levels of $\beta$-gal activity under selective conditions. We also confirmed the interaction of endogenous Rictor with DUSP10 in mammalian cells via co-immunoprecipitation experiments (figure 1B). As shown, anti-DUSP10 antibodies were able to effectively co-immunoprecipitate Rictor, as well as in the reciprocal fashion; Rictor antibodies co-immunoprecipitated DUSP10, while antibodies to the mTORC1 subunit Raptor did not, supporting the notion that DUSP10 is specifically associated with mTORC2. These data suggested that domains within the N-terminal half of Rictor and immediately N-terminal to the MAP kinase binding domain within DUSP10 mediate the interaction of these two proteins and that the full-length endogenous proteins 
Table 1: Genetic interactors identified in yeast two-hybrid screens utilizing Rictor as bait.

\begin{tabular}{|c|c|c|c|}
\hline Prey & Accession \# & \# of hits & Description \\
\hline DUSP 10 & NM_007207 & 32 & Dual specificity phosphatase 10, MKP-5 \\
\hline CUL1 & NM_003592.2 & 10 & Cullin-1 \\
\hline PAK2 & NM_002577.4 & 8 & p21 protein (Cdc42/Rac)-activating kinase 2 \\
\hline AMOTL2 & NM_091278683 & 7 & Angiomotin-like 2 \\
\hline mTOR & NM_004958 & 7 & Target of rapamycin kinase \\
\hline YBX1 & NM_004559 & 6 & Y-box binding protein 1 \\
\hline mLST8 & NM_022372 & 6 & $\mathrm{G}$ protein $\beta$-subunit-like-protein, $\mathrm{G} \beta \mathrm{L}$ \\
\hline SIN1 & NM_0241117 & 5 & $\begin{array}{l}\text { Mitogen-activated protein kinase associate } \\
\text { protein 1, MAPKAP1 }\end{array}$ \\
\hline Hsp70 & NM-005345 & 5 & Heat shock $70 \mathrm{kDa}$ protein $1 \mathrm{~A}$ \\
\hline BAT2 & NM_080686 & 4 & Proline-rich coiled-coil 2A, PRRC2A \\
\hline ILK & NM_001278441 & 3 & Integrin-linked kinase \\
\hline SGK1 & EU518415 & 3 & Serum/glucocorticoid regulated kinase 1 \\
\hline SSH1L & NM_018984.3 & 3 & Slingshot protein phosphatase 1 \\
\hline Protor-1 & NM_001017528 & 2 & Proline rich 5 ORF, PRR5 \\
\hline
\end{tabular}

interact in mammalian cells. Moreover, as purified Rictor did not affect DUSP10 phosphatase activity directly, as determined via an in vitro phosphatase assay (not shown) we hypothesized that DUSP10 may be a substrate for mTORC2 via interaction with Rictor.

\section{DUSP10 is phosphorylated by mTORC2 at serines 224 and 230}

An examination of the DUSP10 sequence identified two potential consensus mTOR phosphorylation sites [22] at serines 224 and 230 within the primary sequence.
We also noted that in glioblastoma cell lines harboring differential mTORC2 activity as a result of ectopic Rictor overexpression [18], DUSP10 mobility was altered in SDS-PAGE analysis. In Rictor overexpressing U87 cells, containing active mTORC2, DUSP10 displayed reduced SDS-PAGE mobility. To determine whether the reduction in DUSP10 mobility was due to phosphorylation we treated cell extracts with lambda PP, which resulted in the elimination of the more slowly migrating species of DUSP10 (figure 2A). Treatment of these cells with PP242 similarly abolished expression of the slower migrating phosphorylated DUSP10 and occurred in a dose-dependent fashion (figure 2A, Supplementary figure
A

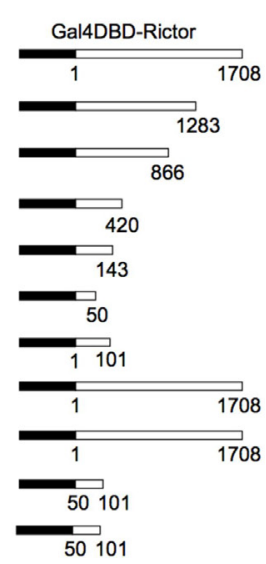

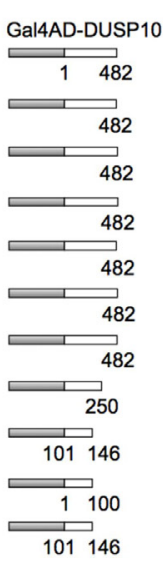

$\begin{array}{cc}\text { growth } & \beta \text {-gal units } \\ +++ & 101 \\ +++ & 142 \\ +++ & 138 \\ ++ & 92 \\ ++ & 101 \\ +- & 11 \\ ++ & 90 \\ ++ & 95 \\ ++ & 93 \\ + & 16 \\ ++ & 97\end{array}$

B

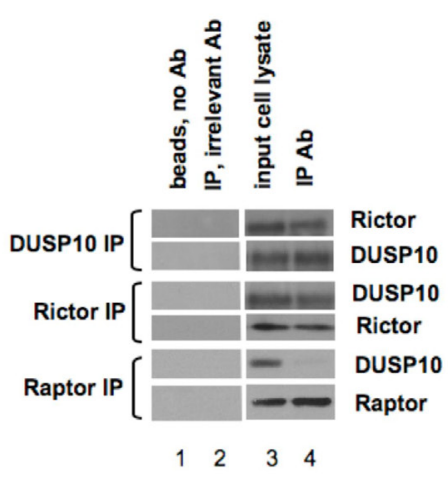

Figure 1: Interaction of Rictor and DUSP10 in yeast and mammalian cells. A). The indicated deletion mutants of Gal4DBDRictor or Gal4AD-DUSP10 were cotransfected into AH109 cells to determine whether an interaction between the proteins was detectable via activation of the HIS3 reporter (+++, strong growth; ++, moderate growth; -, no growth). Colonies which grew were assayed for $\beta$-gal activity. B) Rictor, Raptor or DUSP10 was immunoprecipitated from U87 cells and precipitates subjected to Western analysis for the indicated proteins. Lane 1, beads, no antibody; Lane 2, immunoprecipitation with an irrelevant antibody (control IgG); Lane 3, input cell lysate; Lane 4, indicated immunoprecipitate probed with antibodies for the indicated proteins. As a control, in Raptor immunoprecipitates DUSP10 was not detected. 
S1). Immunoprecipitated mTORC2 phosphorylated recombinant DUSP10 in vitro and the phosphorylation was reversible after addition of lambda PP. These reactions were separated on high-resolution gels to clearly observe the alterations in DUSP10 mobility (figure 2B). Subsequently, we generated substitution mutants of DUSP10 at the candidate mTORC2 phosphorylation sites. Each serine residue was changed to alanine, either individually or in combination. In vitro kinase assays demonstrated that each single DUSP10 mutant exhibited reduced phosphorylation by immunoprecipitated mTORC2 and the double mutant DUSP10 (S224A, S230A), showed no phosphorylation (figure 2C). Moreover, in Rictor overexpressing U87 cells harboring activated mTORC2, the DUSP10 double mutant was not phosphorylated while wild-type DUSP10 displayed significant phosphorylation in vivo (figure 2D). These data demonstrate that $\mathrm{mTORC} 2$ is able to phosphorylate serines 224 and 230 on DUSP10.

\section{Differential mTORC2-dependent stability of DUSP10}

As a major mechanism of DUSP regulation involves regulated degradation via phosphorylation in a proteosome-dependent manner [23], we determined whether modulating mTORC2 activity would result in altered DUSP10 stability. As shown in figure 3A, in the

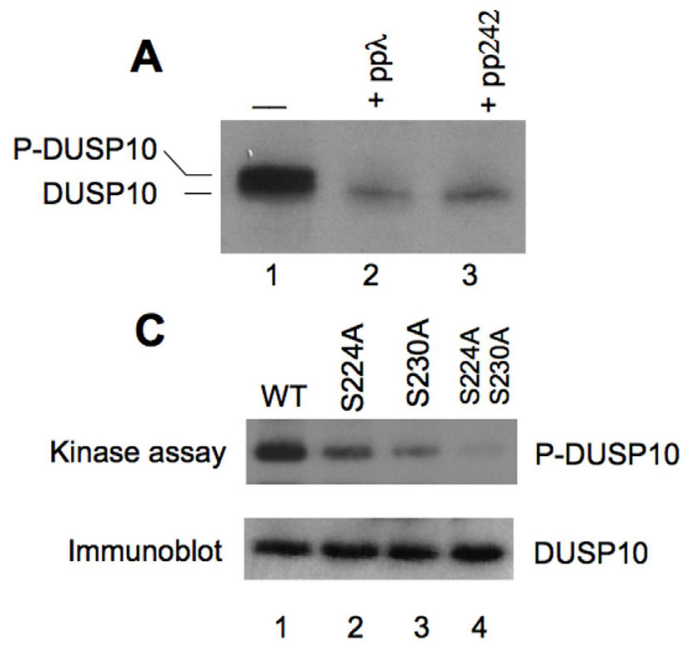

glioblastoma lines U373MG, U87, and LN229 DUSP10 was degraded in a proteosome-dependent manner with a half-life of approximately $90 \mathrm{~min}$, consistent with previous reports of the lability of other DUSPs [5, 24]. However, U87 cells in which ectopic overexpression of Rictor led to increased mTORC2 activity [18], DUSP10 was significantly stabilized $\left(\mathrm{t}_{1 / 2}>3 \mathrm{~h}\right)$ while in cells expressing a shRNA targeting Rictor resulting in loss of mTORC2 activity, DUSP10 was very labile with a calculated halflife of only $30 \mathrm{~min}$ (figure 3B). As shown in figure 3C, DUSP10 was significantly destabilized following PP242 exposure with a calculated half-life of approximately 35 min. Moreover, we confirmed that in DUSP10 knockdown cells p38 MAPK activity is markedly increased, consistent with DUSP10 as being a major negative effector of p38 (figure 3D) [25]. These data suggest that enhanced mTORC2 activity is correlated with a marked increase in DUSP10 protein stability.

\section{Insulin-PI3K signaling stimulates mTORC2 mediated DUSP10 stabilization and p38 inactivation}

Our data thusfar supported a signaling cascade in which activation of mTORC2 and subsequent phosphorylation of DUSP10 would lead to stabilization of the phosphatase and resulting p38 MAPK inactivation.

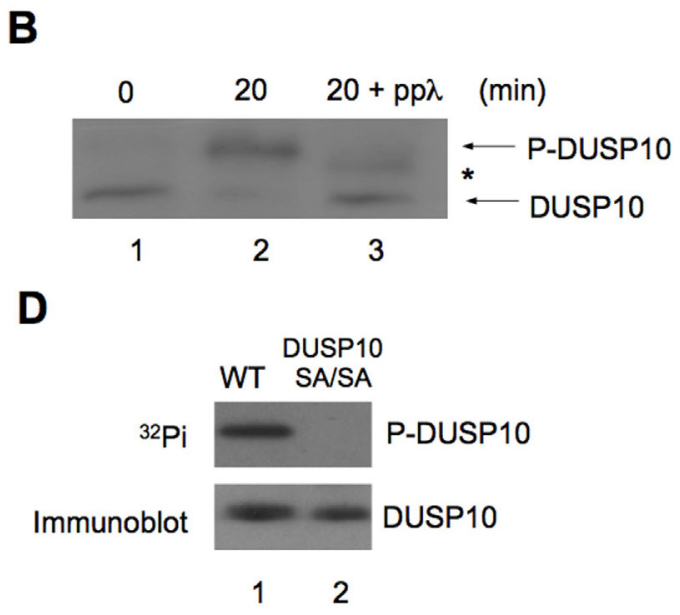

Figure 2: DUSP10 is phosphorylated by mTORC2. A). U87 $7_{\text {Rictor }}$ cells harboring active mTORC2, display a slower migrating DUSP10 species (lane 1) which is eliminated by protein phosphatase lambda ( $\mathrm{pp} \lambda$ ) in vitro (lane 2) or by treating cells with PP242 (50 $\mathrm{nM}$, 24 h) (lane 3). B). Immunoprecipitated mTORC2 phosphorylates recombinant DUSP10 in vitro. High-resolution SDS-PAGE of mTORC2 kinase reactions utilizing recombinant DUSP10 as a substrate and $\left[\gamma^{32} \mathrm{P}\right]$ ATP for the indicated time (min) and treated with pp $\lambda$ as shown. Phosphorylated and unphosphorylated species are as indicated, as well as partially dephosphorylated DUSP10 (asterisk). C). DUSP10 was mutated to produce the single mutants S224A and S230A and the double mutant S224A-S230A and treated with mTORC2 as described in (B). Wild-type (WT) DUSP10 and the mutants were subjected to an in vitro kinase assay with mTORC2 and [ $\left.\gamma^{32} \mathrm{P}\right] \mathrm{ATP}$. Reactions were immunoprecipitated and detected by immunoblotting and autoradiography. D). U87 $7_{\text {Rictor }}$ cells were transfected with expression plasmids encoding DUSP10 or the double mutant S224A-S230A (SA/SA) and $24 \mathrm{~h}$ following transfection cells were labeled with ${ }^{32} \mathrm{P}_{\mathrm{i}}(500 \mu \mathrm{Ci} /$ $\mathrm{ml}$ ) in phosphate-free media for $4 \mathrm{~h}$. DUSP10 was immunoprecipitated, resolved by SDS-PAGE and revealed by autoradiography (top) or immunoblotted (bottom). Results in A, B were performed three times with similar results. 
We next investigated whether DUSP10 protein stability and p38 activity were regulated by insulin. Insulin is known to activate mTORC2 [26] and result in the activation of downstream effectors. Insulin treatment of U87 glioblastoma cells, which had been serumstarved for $18 \mathrm{~h}$, resulted in the significant stabilization of DUSP10 as compared to control, unstimulated cells (figure 4A). Importantly, stabilization of DUSP10 correlated with increased phosphorylation serine 473 on endogenous AKT, a marker of mTORC2 activity (figure 4B). Furthermore, we observed a reduction in DUSP10 mobility and protein accumulation, as well as a marked reduction in phosphorylated p38 levels following insulin treatment consistent with the phosphorylation of DUSP10 by $\mathrm{mTORC} 2$ and resultant dephosphorylation of $\mathrm{p} 38$. These data demonstrate that insulin stabilizes DUSP10.

Insulin is known to activate mTORC2 via PI3K [27]. To ascertain whether PI3K signaling regulates DUSP10 stability, we examined whether DUSP10 degradation was affected upon blockade or stimulation of PI3K signaling. Inhibition of PI3K/mTORC2 signaling in cells treated with PI3K inhibitor LY294002 had no significant affect on DUSP10 stability and resulted in inhibition of phosphoSer ${ }^{473}-\mathrm{AKT}$ and enhanced phospho-Thr ${ }^{180} / \mathrm{Tyr}^{182}$-p38 levels (figure 4C). DUSP10 SDS-PAGE mobility was also consistent with migration of the non-phosphorylated form of the phosphatase. In contrast, hyperactivation of PI3K signaling, accomplished by PTEN knockdown, a known negative regulator of PI3K signaling [28], markedly stabilized DUSP10 which accumulated to high levels in its phosphorylated form as compared to control cells (figure 4D). Again, the stabilization of DUSP10 upon PI3K hyperactivation was mirrored by a concomitant increase in the phosphorylation status of the endogenous mTORC2 substrate $\mathrm{Ser}^{473}$-AKT. Taken together these data suggest that insulin stimulates DUSP10 phosphorylation and stabilization in a physiological relevant manner dependent on PI3K/mTORC2 signaling.
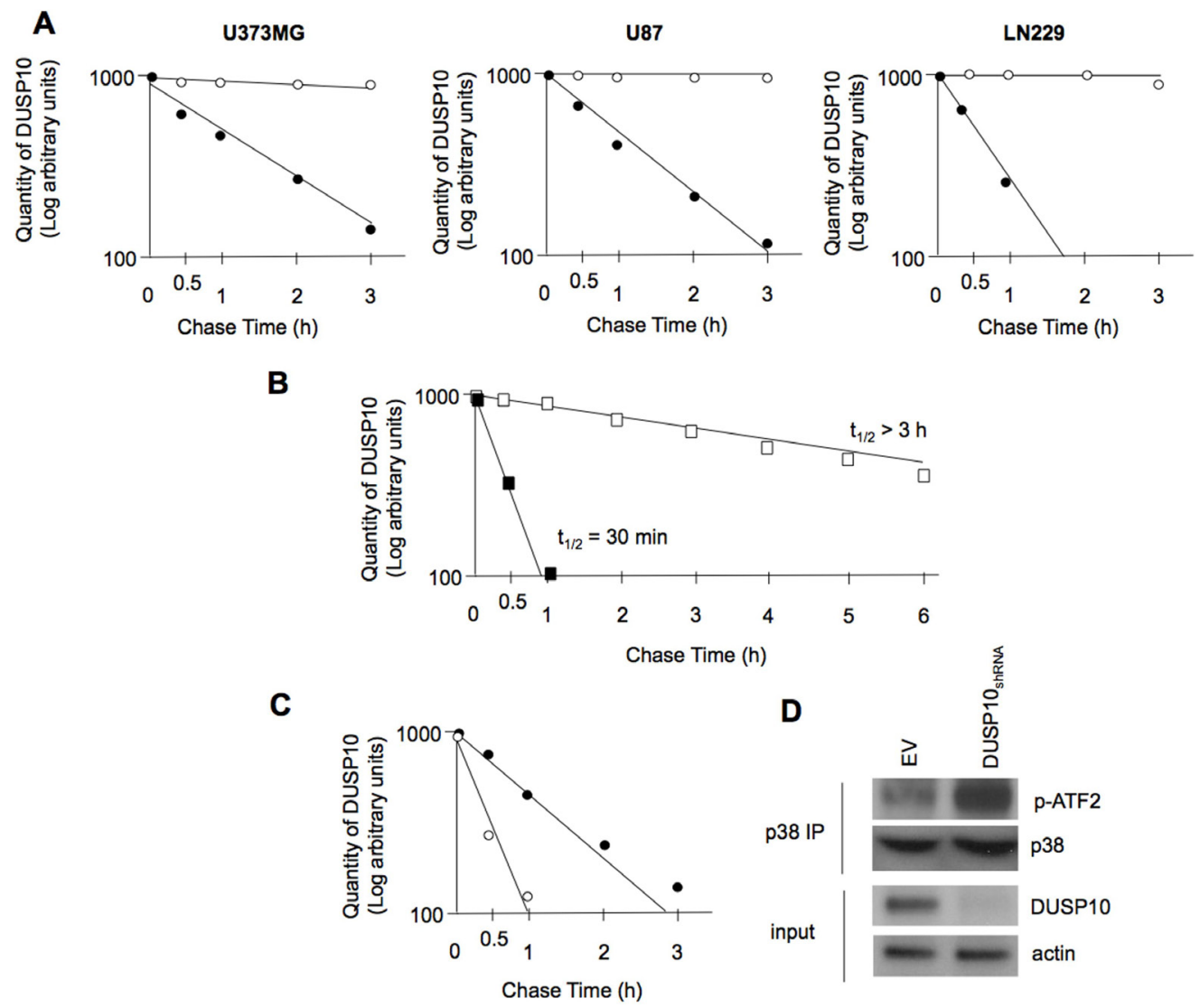

Figure 3: Half-life of DUSP10 is altered in response to modulation of mTORC2. A). Basal half-life of DUSP10 in U373MG (left panel), U87 (middle panel) and LN229 (left panel) glioblastoma cells. Cells were pulsed with ${ }^{35}$ S-methionine and DUSP10 levels monitored as described in the Methods section. Solid circles are in the absence, while open circles are in presence of the proteosome

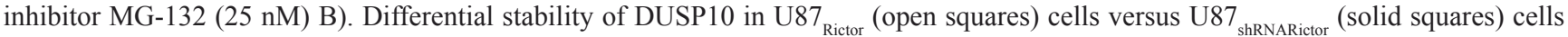
[18]. C). Destabilization of DUSP10 following PP242 exposure (10 nM, $6 \mathrm{~h}$ ) in U373MG cells. Solid circles are in the absence of PP242, while open circles are in the presence of PP242. D). DUSP10 knockdown in U87 glioblastoma cells leads to enhanced p38 activity. Lysates from cells expressing shRNA targeting DUSP10 or empty vector (EV) were immunoprecipitated for p38 and immunoprecipitates subjected to an in vitro kinase assay using ATF2 as a substrate. Phosphorylated ATF2 was detected using phosphospecific antibodies. Input lysates were immunoblotted for the indicated proteins. 


\section{RNAi-mediated DUSP10 knockdown or overexpression of DUSP10 S224/S230 mutants alters responses of GBM cells to mTOR kinase inhibitors}

Having previously demonstrated that a major mode of mTOR inhibitor resistance in GBMs was dependent on the induction p38 MAPK signaling [21] and to expand on the possible functional significance of mTORC2-mediated phosphorylation of DUSP10, we initially examined whether the U87 line expressing the shRNA targeting DUSP10 (figure 3D) displayed an altered response to
PP242 as compared to empty vector control transfected cells. As shown in figure 5A, cells in which DUSP10 had been knocked-down were significantly more resistant to PP242 as compared to controls as determined by an XTT proliferation assay. In addition, the percentage of apoptotic cells, determined by Annexin V staining, markedly decreased from control values (percent apoptotic cells shown above bars). As expected, knockdown of DUSP10 or, as previously observed, following PP242 exposure, resulted in elevated phospho-p38 levels (figure 5B). Moreover, PP242 treatment effectively inhibited mTORC2 activity as phospho-AKT levels were dramatically reduced. These results supported our previous observations

A

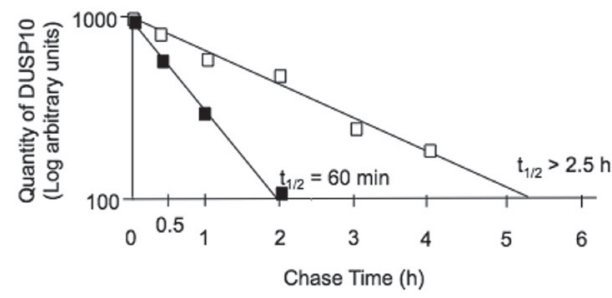

B

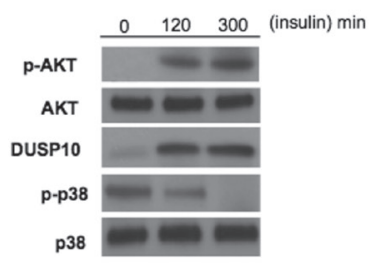

C
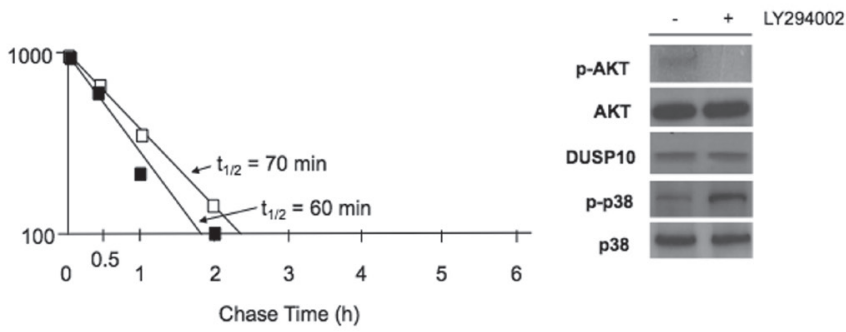

D
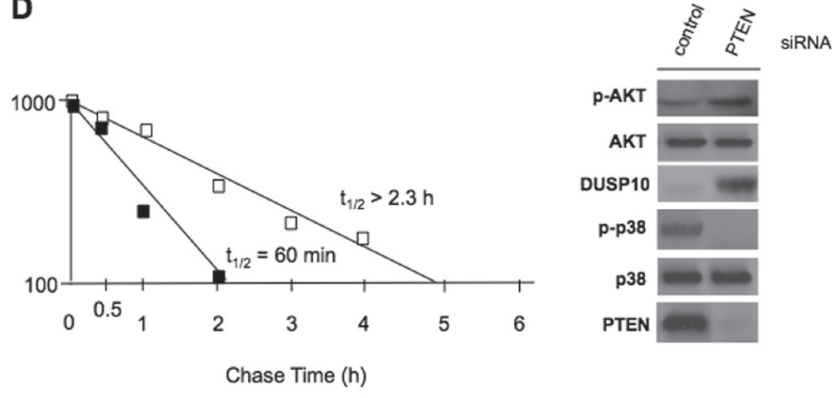

Figure 4: Insulin-PI3K signaling regulates DUSP10 stability and phosphorylated p38 levels. A) Half-life of DUSP10 in U87 cells under basal (solid squares) and following insulin stimulation (open squares). B). Signaling effects of insulin stimulation in U87 cells. Insulin-stimulated cells $(10 \mathrm{nM})$, treated for the indicated time points, were lysed and extracts immunoblotted for the indicated proteins. C). DUSP10 half-life (left panel) and signaling (right panel) in U87 cells treated with LY294002 (50 $\mu \mathrm{M})$ (untreated, solid squares; LY294002 treated, open squares). D). siRNA-mediated knockdown of PTEN in LN229 cells leads to stabilization of DUSP10 (left panel; untreated solid squares; PTEN siRNA treated, open squares). Extracts from cells transfected with the indicated siRNA and immunoblotted for the indicated proteins (right panel). 
that p38 MAPK activity mediated a resistance pathway to mTOR inhibitors in GBMs [21] and were consistent with the notion that negating the DUSP10 blockade to p38 activity should result in a resistant cell phenotype in the face of PP242 exposure. Subsequently, we examined whether forced overexpression of nonphosphorylatable $(224 \mathrm{~A} / 230 \mathrm{~A})$ or phosphomimetic $(224 \mathrm{E} / 230 \mathrm{E})$ mutants as compared to wild-type DUSP10, or empty vector controls, would have effects on PP242 sensitivity. As shown in figure 5C, XTT proliferation assays demonstrated that U87 GBM cells stably overexpressing DUSP10 224A/230A were significantly more resistant to PP242 as compared to cells overexpressing wild-type DUSP10, however we also observed that native DUSP10 significantly decreased resistance to the drug upon overexpression. Additionally, the number of apoptotic cells was significantly reduced in PP242-treated cells overexpressing the nonphosphorylatable mutant, while cells overexpressing
A

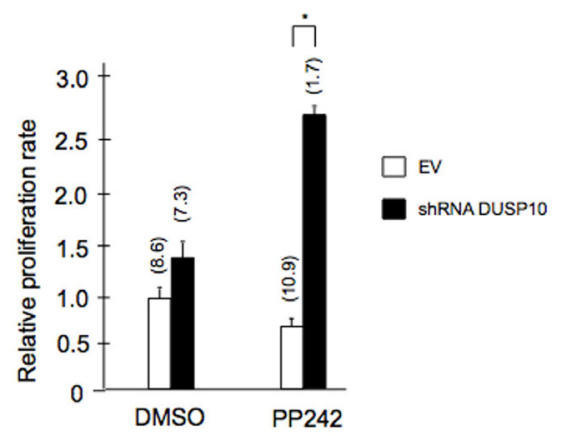

C

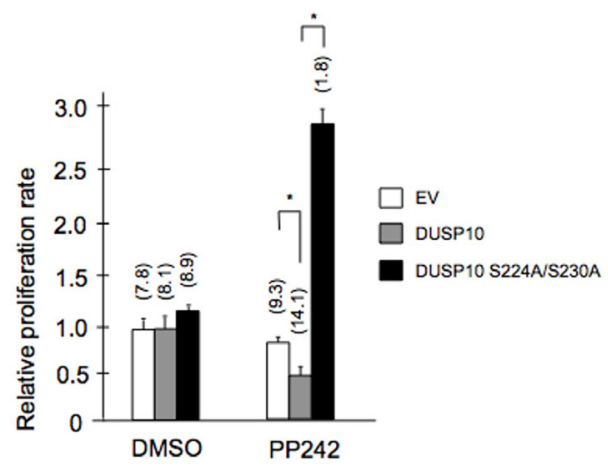

$\mathbf{E}$

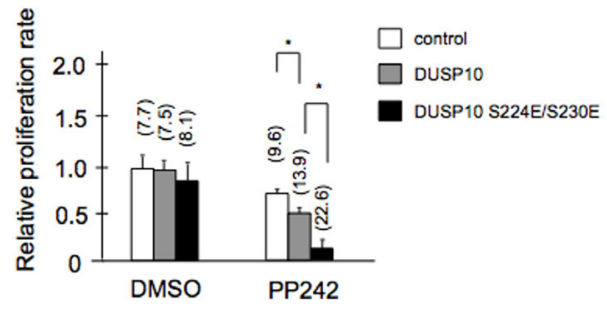

B

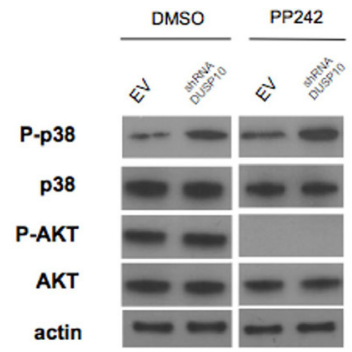

D

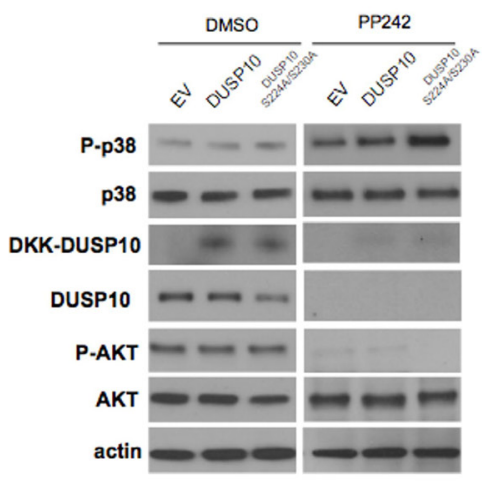

$\mathbf{F}$

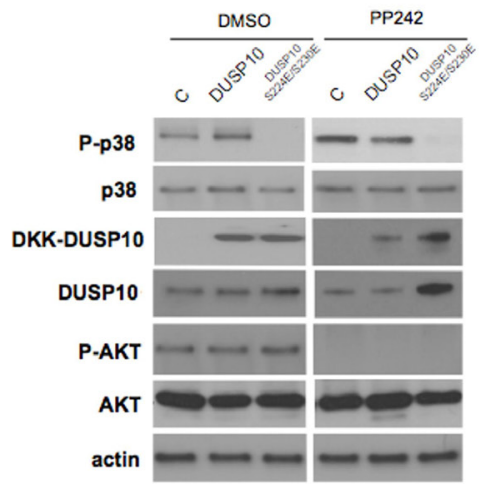

Figure 5: Effects of DUSP10 knockdown and nonphosphorylatable or phosphomimetic mutant overexpression on GBM responses to mTOR kinase inhibition. A) Relative proliferation rates as determined from XTT proliferation assays of U87 cells expressing shRNA targeting DUSP10 as compared to empty vector (EV) transfected cells in the presence or absence of PP242 (25 $\mathrm{nM}, 24 \mathrm{~h}$ ). Values in parentheses above bars correspond to percent apoptotic cells as determined via Annexin $\mathrm{V}$ staining. ${ }^{*} P<0.05$ as determined by Student's $t$-test. B) Relative p38 and mTORC2 signaling in cells from (A). Extracts were prepared from the indicated lines and treatment groups and subjected to immunoblot analysis for the proteins shown. C\&D) Effects of PP242 on proliferation, apoptosis and signaling in U87 cells overexpressing nonphosphorylatable DUSP S224A/S230A. ${ }^{*} P<0.05$. E\&F) As in (C\&D), except performed using cells overexpressing the phosphomimetic DUSP10 S224E/S230E mutant. * $P<0.05$. 
native DUSP10 displayed a modest increase in annexin V-positive cell numbers. The nonphosphorylatable DUSP10 mutant expressing cells also displayed elevated levels of phosphorylated p38 and downregulated DUSP 10 expression, relative to empty vector alone-transduced cells or cells overexpressing native DUSP10 (figure 5D). Overexpression of the DUSP10 mutants was confirmed by immunoblotting for the DKK-tagged allele and a marked reduction in phospho-AKT levels in PP242-treated cells was observed demonstrating ablation of mTORC2 activity. DUSP10 levels were not detectable in PP242-treated lines consistent with the inhibition of mTORC2 activity. In U87 cells stably overexpressing the DUSP10 phosphomimetic mutant (224SE/230SE) we observed a significant increase in sensitivity to PP242 as compared to controls (figure 5E). PP242-induced apoptosis also increased markedly in the phosphomimetic overexpressing cells. As shown in figure $5 \mathrm{~F}, \mathrm{p} 38$ signaling was blunted in cells overexpressing the phosphomimetic mutant, which correlated with relative increases in the levels of detectable total DUSP10. We also determined the relative half-lives of both the nonphosphorylatable and phosphomimetic DUSP10 proteins and as shown in supplementary figure S2, these were comparable to the half-lives observed for endogenous DUSP10 in lines containing quiescent or elevated mTORC2 activity, respectively (see figure 3C). Moreover, the calculated half-life observed for endogenous DUSP 10 following PP242 treatment was comparable to that which was seen for the phosphomimetic mutant under similar conditions (see figure $3 \mathrm{C}$ ). These data confirm a specific role for DUSP10 and support the functional significance of mTORC2-mediated phosphorylation events in determining the response to mTOR kinase inhibitors.

\section{In vivo effects of PP242 on mutant DUSP10 expressing lines}

To ascertain what effects PP242 would have on the DUSP10 mutant overexpressing cell lines in vivo we performed xenografts experiments in SCID mice. Parental U87, U87-DUSP10 S224A/S320A (nonphosphorylatable) and U87-DUSP10 S224E/S230E (phosphomimetic) expressing lines were used to establish subcutaneous tumors in the rear flanks of mice and upon reaching $\sim 200$ $\mathrm{mm}^{3}$ in size were treated with either vehicle or PP242 for 10 consecutive days beginning at day 0 (figure 6A-C). In tumor cells overexpressing the nonphosphorylatable DUSP10 mutant xenografts, growth was modestly enhanced by PP242 treatment. Apoptosis induction in these tumors, as monitored by TUNEL staining following the 10-day treatment course, also showed a modest yet significant decrease relative to vehicle treated mice (figure 6D). P38 signaling in these tumors was significantly induced following PP242 treatments while DUSP10 expression was reduced (figure 6E). Conversely, in tumors derived from the phosphomimetic DUSP10 overexpressing cells, PP242 treatment markedly reduced tumor size, increased apoptotic induction, blunted p38 signaling and increased DUSP10 expression. These data support the in vitro effects observed for DUSP10 in response to PP242 and confirm the importance of the DUSP10 S244 and S230 phosphorylations on mTOR kinase inhibitor responses in vivo.

\section{DUSP10 is overexpressed in GBM patients}

To seek clinical validation of the signaling relationships observed, we determined whether DUSP10 expression was altered in GBM. We analyzed an independent set of 32 quick-frozen glioblastoma samples and 5 normal samples. Each tumor sample was confirmed histologically, extracts prepared and the total relative abundance of DUSP10, phospho-Ser- ${ }^{473}$-AKT and phospho-Thr ${ }^{180} / \mathrm{Tyr}^{182}-\mathrm{p} 38$ expressed determined by Western analysis. Additionally, we determined the phosphorylation status of DUSP10 via high-resolution SDS-PAGE mobility as before. As shown in Table 2, seventeen of $32(53 \%)$ tumor samples exhibited markedly elevated DUSP10 expression, which we defined as greater then a 10-fold increase relative to mean levels expressed in normal brain. We also observed a significant direct correlation in samples containing elevated DUSP10 levels with increased mTORC2 activity as well as phosphorylation of DUSP10 $(P<0.01)$. Moreover, elevated DUSP10 expression was significantly inversely correlated with p38 activity $(P<0.01)$. These data indicate that DUSP10 protein levels and phosphorylation is increased in GBM tumors and support the signaling pathways delineated in the cell line experiments.

\section{DISCUSSION}

The activation of MAP kinases is accomplished by phosphorylation of both threonine and tyrosine residues within the signature sequence TXY of the kinase domain VIII [1]. The upstream activators have been identified and characterized and these are specific for each class of MAP kinase [29]. These kinases are inactivated by the dephosphorylation of these residues, which are carried out by members of the dual-specificity phosphatases [3, 23]. Certain DUSPs are inducible, while others are stabilized or destabilized by phosphorylation. Phosphorylation of DUSPs can lead to their stabilization by attenuating ubiquitination and subsequent degradation. For example, phosphorylation via ERK stabilizes DUSP1 and increases its half-life, whereas it elicits DUSP3 degradation [5, 
Table 2: Relative DUSP10 expression and phosphorylation status in 32 primary glioblastoma samples.

\begin{tabular}{|c|c|c|c|c|}
\hline Samples & \begin{tabular}{|l|} 
Relative DUSP10 \\
expression§
\end{tabular} & $\begin{array}{l}\text { Phosphorylated } \\
\text { DUSP10 }\end{array}$ & $\begin{array}{l}\text { Relative mTORC2 } \\
\text { activity }^{\wedge}\end{array}$ & $\begin{array}{l}\text { Relative p38 activ- } \\
\text { ity } ¥\end{array}$ \\
\hline \multicolumn{5}{|l|}{ Normal } \\
\hline 1 & 1.3 & - & 1.4 & 1.2 \\
\hline 2 & 1.1 & - & $-\dagger$ & $-t$ \\
\hline 3 & 1.3 & - & 1.5 & 1.1 \\
\hline 4 & 1.2 & - & 1.3 & $\begin{array}{ll}1.7 \\
\end{array}$ \\
\hline 5 & 1.1 & - & 1.6 & 1.2 \\
\hline \multicolumn{5}{|l|}{ GBM } \\
\hline 1 & $54.9 *$ & + & 39.7 & 10.6 \\
\hline 2 & 1.1 & - & 0.1 & 26.4 \\
\hline 3 & $60.2^{*}$ & + & 34.9 & 2.6 \\
\hline 4 & $82.4^{*}$ & + & 23.2 & 5.1 \\
\hline 5 & 5.6 & - & 0.5 & 39.7 \\
\hline 6 & $45.9 *$ & + & 63.6 & 4.1 \\
\hline 7 & 9.2 & - & 1.7 & 40.6 \\
\hline 8 & $50.6^{*}$ & + & 25.4 & 2.0 \\
\hline 9 & $40.1^{*}$ & + & 55.3 & - \\
\hline 10 & $35.2 *$ & $\begin{array}{lll}+ & \\
\end{array}$ & 17.7 & 3.7 \\
\hline 11 & $56.3^{*}$ & + & 26.0 & 0.9 \\
\hline 12 & 3.3 & - & 5.3 & 19.2 \\
\hline 13 & $29.6^{*}$ & + & 41.2 & 2.7 \\
\hline 14 & 6.7 & - & 5.7 & 26.4 \\
\hline 15 & 2.3 & - & 1.0 & 35.8 \\
\hline 16 & $17.9^{*}$ & + & 19.4 & - \\
\hline 17 & 5.1 & - & 0.7 & 17.1 \\
\hline 18 & 2.1 & - & - & 12.9 \\
\hline 19 & $29.4 *$ & + & 37.6 & 0.1 \\
\hline 20 & 1.4 & - & 0.3 & 36.3 \\
\hline 21 & $30.5^{*}$ & + & 17.9 & 10.2 \\
\hline 22 & 0.8 & - & 4.3 & 22.8 \\
\hline 23 & $43.1 *$ & + & 45.0 & 9.5 \\
\hline 24 & $27.1^{*}$ & + & 19.6 & 2.2 \\
\hline 25 & 0.7 & - & 0.6 & 17.9 \\
\hline 26 & $71.6^{*}$ & + & 22.9 & 6.5 \\
\hline 27 & $25.3^{*}$ & + & 16.6 & 11.5 \\
\hline 28 & 5.1 & - & 3.8 & 11.7 \\
\hline 29 & $47.1^{*}$ & + & 19.4 & 0.8 \\
\hline 30 & 2.8 & - & 0.2 & 29.7 \\
\hline 31 & 4.7 & - & 2.9 & 19.6 \\
\hline 32 & 0.9 & - & 1.3 & 26.2 \\
\hline
\end{tabular}

Note: Five normal brain and 32 quick-frozen GBM samples were assessed for DUSP10 expression and phosphorylation as described in figure 2 and quantified. Seventeen of 32 tumor samples (53\%) had markedly higher expression levels of DUSP10 and displayed migration patterns consistent with phosphorylated DUSP10 $(+)$ relative to normal brain. mTORC2 and p38 activity was determined by monitoring phospho-Ser ${ }^{473}$-AKT and phospho-Thr ${ }^{180} /$ $\mathrm{Tyr}^{182}$-p38, respectively.

$\dagger$ Undetectable phospho-Ser ${ }^{473}-\mathrm{AKT}$

+ Undetectable phospho-Thr ${ }^{180} / \mathrm{Tyr}^{182}-\mathrm{p} 38$.

$\S$ DUSP10 expression $>2$-fold above mean of normal brain.

* Markedly increased DUSP10 expression > 10-fold increase above mean of normal brain.

mTORC2 activity $>2$-fold above mean of normal brain.

$¥$ p38 activity $>2$-fold above mean of normal brain. 
30]. Our work supports the hypothesis that mTORC2 phosphorylates DUSP10 resulting in its stabilization. While we cannot formally exclude the possibility that additional kinases may similarly phosphorylate DUSP10 leading to stabilization, this stabilization presumably leads to accumulation of the protein which promotes its ability to dephosphorylate and inactivate $\mathrm{p} 38$. As our previous studies implicated sustained activation of the $\mathrm{p} 38$ signaling pathway in mTOR inhibitor resistance [21], we further tested whether alterations in DUSP10 activity would affect PP242 GBM tumor cell responses. Our data also support the contention that mTOR kinase inhibition, with compounds such as PP242, disrupt the ability of mTORC2 to stabilize DUSP10, leading to its rapid turnover with a resultant increase in p38 signaling activity.

Previous data supports the involvement of the mTOR signaling pathway in the regulation of the ERK phosphatase DUSP6 [31]. ERK/mTOR phosphorylation of DUSP6 on serine 159 and ERK phosphorylation on serine 197 induced a significant reduction in DUSP6 half-life promoting a positive feedback loop in response to MAPK signaling [32]. In contrast, our data show that phosphorylation of DUSP 10 via mTORC2 on serines 224 and 230 enhances DUSP 10 protein stability and negatively regulates p38 activity in response to PI3K activation. These variations in DUSP protein stability highlight the complex regulation of DUSPs post-translationally and may be a result of distinct signaling pathway inputs,
A

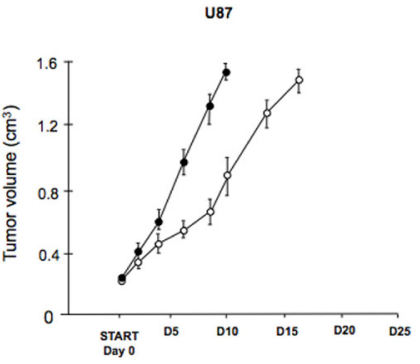

C

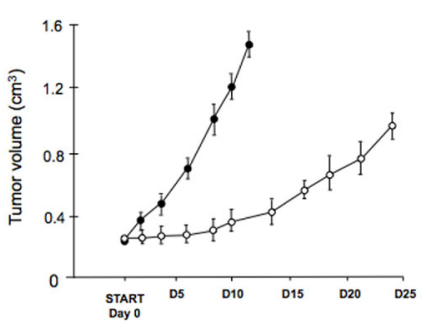

B

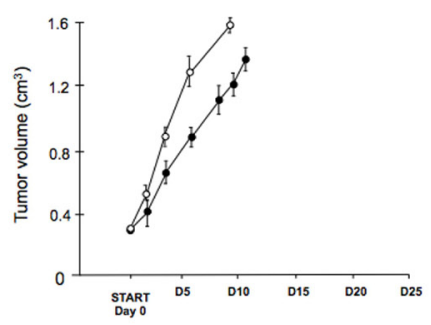

D

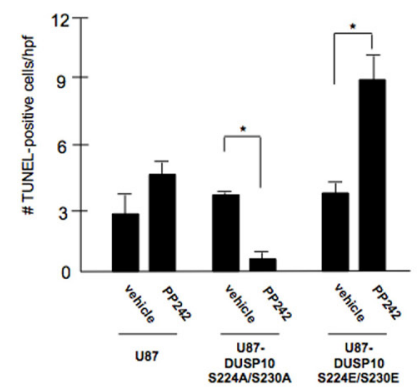

E
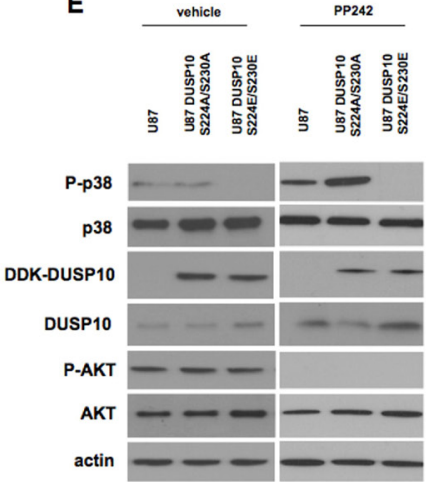

Figure 6: In vivo effects of PP242 on DUSP10 nonphosphorylatable or phosphomimetic expressing GBM lines. A-C). Effects of PP242 treatment on U87, U87 DUSP10 S224A/S230A or U87 DUSP10 S224E/230E expressing lines in xenografts as indicated. Mice with established tumors $\left(200 \mathrm{~mm}^{3}\right)$ received either vehicle (closed circles) or PP242 $(20 \mathrm{mg} / \mathrm{kg} / \mathrm{day}$, open circles) for 10 consecutive days and tumor growth was assessed every 2 days following initiation of treatment (start, day 0). D). Quantification of in situ TUNEL assay results from xenografts harvested at day 10 as indicated. The data are expressed as the number of positive apoptotic bodies divided by high power field (hpf; 10-12 hpf/tumor). Values are the means + SD. $* P<0.05$. E). Effects of PP242 therapy on signaling pathways from day 10 harvested xenografts from the indicated lines. Immunoblot analysis was performed for the indicated proteins. 
particularly in the above case, as mTOR phosphorylation of DUSP6 was shown to be rapamycin sensitive and thus likely to be mediated by mTORC1.

While our data support the regulation of DUSP10 turnover by MTORC2 we cannot rule out the possibility that phosphorylation at serines 224 and 230 also may have effects on substrate targeting. Although we observed nearly complete dephosphorylation of p38 following mTORC2-mediated phosphorylation of DUSP10 following knockdown of PTEN (see figure 4D), which is consistent with the substrate preference of DUSP10 for p38 relative to other MAPKs, these residues are within the kinase binding domain of DUSP10 and may alter the affinity of the MKP-MAPK interaction. Indeed, Dickinson et al., demonstrated that serine 58 phosphorylation within the kinase interaction motif of DUSP9 impaired its ability to interact with ERK and p38 [33]. Interestingly, acetylation of lysine 57 of DUSP1, which is also within close proximity to critical arginine residues within its kinase interaction motif, stimulated binding of p38 [34]. It is certainly possible that the mTORC2-mediated phosphorylation events on DUSP10 or additional posttranslational modifications are able to regulate substrate specificity.

It has been observed that individual DUSPs may have either increased or decreased expression in different tumor types [23]. For example, some DUSPs exhibit loss of heterozygosity in some cancers with additional functional studies supporting potential roles as tumor suppressors, including DUSP4, DUSP6 and DUSP7 [3537]. However, some DUSPs are associated with increased expression in cancer progression. For example, DUSP1 expression is increased in pancreatic cancer and shown that its downregulation leads to reduced tumorigenicity [38]. Additionally, DUSP6 has been shown to be upregulated in non-small-cell lung carcinomas exhibiting hyperactive receptor tyrosine kinase activity and Ras/ Raf signaling and potentially functioning as a negativefeedback regulator of mitogenic signaling [39]. Our data demonstrate that DUSP10 is overexpressed in a significant number of GBM samples and whether it plays a role in gliomagenesis and/or progression will be the subject of future experiments. Additional studies have also linked DUSP activity to chemotherapeutic resistance [30]. DUSP1 has been demonstrated to sensitize cancer cells to cisplatin-induced apoptosis [40] and overexpression of DUSP6 in estrogen receptor-positive breast cancer cells confers resistance to tamoxifen [41]. However, loss of DUSP6 expression in ovarian cancer cells has been demonstrated to increase chemoresistance [42]. Our RNAi-mediated DUSP10 knockdown and S224/S230 mutant overexpression data clearly demonstrate that reduced DUSP10 expression/activity results in heightened resistance to mTOR kinase targeted therapy in GBM lines, while an increase in DUSP10 expression/activity had the opposing effect (see figure 5).

In conclusion, we have identified DUSP10 as a novel substrate for mTORC2 via its interaction with Rictor. mTORC2-mediated phosphorylation of DUSP10 resulted in significant stabilization of the phosphatase and correlated with inhibition of p38 activity. Moreover, modulating DUSP10 activity resulted in altered GBM tumor responses to PP242 and elevated DUSP10 expression was demonstrated in a significant number of GBM patient tumors. Our data would also predict sensitivity to mTOR kinase inhibitors of GBM tumors harboring elevated DUSP10 expression. Given the importance of mTOR and DUSP/MKP signaling in tumor growth and drug resistance, a further detailed understanding of the signaling relationships linking these two pathways is warranted to effectively target these signaling cascades.

\section{MATERIALS AND METHODS}

\section{Plasmids, cell lines, GBM samples and reagents}

U87 and LN229 cell lines were obtained from ATCC (Manassas, VA). U373MG cells were obtained from Sigma. DNA constructs composed of portions of Rictor and DUSP10 were generated by PCR and individually subcloned into pGB12 and pACT2, respectively. Antibodies to Rictor, Raptor, mTOR, phospho-Akt (Ser473), Akt, DUSP10, p38, phospho-p38 (Thr180/ Tyr182) and phospho-ATF-2 (Thr71) were from Cell Signaling (Beverly, MA). Actin and rabbit IgG (isotype control) antibodies were from Santa Cruz Biotechnology. PTEN antibodies and protein phosphatase lambda were from Millipore. DKK antibodies were from Origene. All other reagents were obtained from Sigma. To generate the DUSP10 S224/S230 substitution mutants the full length DUSP10 cDNA cloned into pCMV6-Entry (OriGene Technologies) was mutagenized using the QuikChange ${ }^{\circledR}$ II Site-Directed Mutagenesis kit (Agilent Technologies) utilizing appropriate mutagenic primers according to the manufacturer. All plasmids were sequenced to verify the constructs. Recombinant wild-type and mutant DUSP10 was expressed and purified from HEK293 cells using anti-FLAG immunoaffinity column chromatography as previously described [15]. DUSP10 shRNA constructs were also obtained from OriGene cloned into the retroviral vector pRS. Cells stably expressing the knockdown constructs were selected via culture in puromycin following retroviral transduction according to the manufacture's protocol. siRNA targeting PTEN was obtained from Cell Signaling (SignalSilence ${ }^{\circledR}$ PTEN siRNA I). Transfections were performed using Effectene ${ }^{\circledR}$ 
transfection reagent according to the manufacturer (Qiagen). Flash-frozen normal brain and glioblastoma samples were obtained from the Cooperative Human Tissue Network (CHTN), National Cancer Institute (Western Division, Vanderbilt University Medical Center) under an approved Institutional Review Board protocol.

\section{Yeast two-hybrid analysis}

The yeast two-hybrid assays to isolate and map Rictor interacting proteins and domains were performed using standard procedures [16]. The full-length human Rictor cDNA was cloned into pGB12 in frame with the Gal4 DNA-binding domain. This construct was used to transform AH109 cells to obtain a strain which expressed the GAL4DBD-Rictor fusion. This strain was used to screen human brain cDNA libraries prepared from mRNA isolated from U87 and T98G cells (lines overexpressing Rictor), which was reverse-transcribed, size-selected and cloned into pACT2 (BD Biosciences, Clontech). Liquid $\beta$-gal assays were performed as previously described [16].

\section{Immunoprecipitations and protein analysis}

Immunoprecipitations and Western analyses were performed as previously described [17], except that TrueBlot ${ }^{\mathrm{TM}}$ reagents (Rockland) were used for DUSP10 immunoprecipitations to avoid IgG heavy chain masking. For DUSP10 half-life determinations, cells were cultured with ${ }^{35} \mathrm{~S}$-methionine for $45 \mathrm{~min}$ followed by the addition of unlabeled methionine $(1 \mu \mathrm{M})$. DUSP 10 was immunoprecipitated at the indicated time points and imaged via autoradiography. DUSP10 bands were excised and counted in a scintillation counter for quantification. Data shown are the means from three independent experiments.

\section{In vitro kinase and phosphatase assays}

mTORC2 in vitro kinase assays were performed as previously described [18] except performed in the presence of $50 \mu \mathrm{M}$ ATP and $\left[\gamma^{32} \mathrm{P}\right] \mathrm{ATP}$ [ $2 \mu \mathrm{Ci}$ per sample] together with $2 \mathrm{ng}$ of in vitro transcribed and translated wild-type or mutant DUSP10 as indicated. Reactions were terminated by addition of $1 \%$ SDS and $4 \mathrm{M}$ urea and resolved by SDS-PAGE. p38 in vitro kinase assays were performed as described [19], utilizing recombinant ATF2 as a substrate and phosphorylation determined via immunoblotting with phospho-specific antibodies. DUSP10 phosphatase activity assays were performed using O-methyl fluorescein phosphate (OMFP) as previously described [20].

\section{Cell proliferation/apoptosis assays}

Cells were seeded in 96-well plates and were treated with mTOR kinase inhibitor for $24 \mathrm{~h}$ as indicated in media containing $1 \%$ FBS. Relative proliferation to control and empty vector transfected cells with vehicle treatment was determined via an XTT Cell Proliferation Assay Kit II (Roche Diagnostics). Cells were incubated for $2 \mathrm{~h}$ following the addition of the tetrazolium salt XTT at $5 \% \mathrm{CO} 2$ and $37^{\circ} \mathrm{C}$ and absorbances of control DMSO or PP242 treated cells was measured with a microplate reader (Bio-Rad) at 420-480 $\mathrm{nm}$. Cells were stained for Annexin $\mathrm{V}$ using a fluorescein isothiocyanate-conjugated anti-Annexin $\mathrm{V}$ antibody (Abcam) and analyzed via flow cytometric measurements. For terminal deoxynucleotidyl transferase-mediated dUTP nick end labeling (TUNEL) staining of sections, slides were stained using the TACS$\mathrm{XL}^{\circledR}$ DAB In situ Apoptosis Detection kit (Trevigen) according to the manufacturer's instructions and counterstained with hematoxylin as previously described [21].

\section{In vivo studies}

For murine xenografts experiments $1 \times 10^{6}$ cells of the indicated lines were injected subcutaneously into the right flank of 4-wk-old SCID mice in $100 \mu \mathrm{l}$ of Matrigel $^{\mathrm{TM}}$, PBS 1:2 solution. After tumors reached $\sim 200 \mathrm{~mm}^{3}$, mice were treated daily with $20 \mathrm{mg} / \mathrm{kg}$ PP242 for 10 days and tumors measured with an electronic caliper and volumes calculated using the formula $L \times W^{2} \times 0.5$, where $L$ is the longest length, and $W$ is the shortest length. Mice were euthanized after when tumor volumes of different lines reached statistical significance. Tumors were also harvested for immunoblot analyses of relevant signaling proteins. Mice were euthanized in accordance with approved institutional guidelines for animal welfare.

\section{ACKNOWLEDGMENTS}

We thank Drs. Alan Lichtenstein, Robert Nishimura and Richard Weisbart for comments on the manuscript. We also thank Jonathan $\mathrm{Li}$ and Lindsay Cooper for technical assistance. This study was supported, in part, by funds from the Veterans Administration and NIH grant R01CA16870.

\section{REFERENCES}

1. Cargnello M and Roux PP. Activation and function of the MAPKs and their substrates, the MAPK-activated protein kinases. Microbiol Mol Biol Rev. 2011; 75(1):50-83.

2. Raingeaud J, Gupta S, Rogers JS, Dickens M, Han J, Ulevitch RJ and Davis RJ. Pro-inflammatory cytokines and environmental stress cause p38 mitogen-activated protein 
kinase activation by dual phosphorylation on tyrosine and threonine. J Biol Chem. 1995; 270(13):7420-7426.

3. Huang CY and Tan TH. DUSPs, to MAP kinases and beyond. Cell Biosci. 2012; 2(1):24.

4. Todd JL, Tanner KG and Denu JM. Extracellular regulated kinases (ERK) 1 and ERK2 are authentic substrates for the dual-specificity protein-tyrosine phosphatase VHR. A novel role in down-regulating the ERK pathway. J Biol Chem. 1999; 274(19):13271-13280.

5. Brondello JM, Pouyssegur J and McKenzie FR. Reduced MAP kinase phosphatase-1 degradation after p42/ p44MAPK-dependent phosphorylation. Science. 1999; 286(5449):2514-2517.

6. Laplante M and Sabatini DM. mTOR signaling in growth control and disease. Cell. 2012; 149(2):274-293.

7. Wullschleger S, Loewith R and Hall MN. TOR signaling in growth and metabolism. Cell. 2006; 124(3):471-484.

8. Feldman ME, Apsel B, Uotila A, Loewith R, Knight ZA, Ruggero D and Shokat KM. Active-site inhibitors of mTOR target rapamycin-resistant outputs of mTORC1 and mTORC2. PLoS Biol. 2009; 7(2):e38.

9. Ma XM and Blenis J. Molecular mechanisms of mTORmediated translational control. Nat Rev Mol Cell Biol. 2009; 10(5):307-318.

10. Sarbassov DD, Guertin DA, Ali SM and Sabatini DM. Phosphorylation and regulation of $\mathrm{Akt} / \mathrm{PKB}$ by the rictormTOR complex. Science. 2005; 307(5712):1098-1101.

11. Hung CM, Garcia-Haro L, Sparks CA and Guertin DA. mTOR-dependent cell survival mechanisms. Cold Spring Harb Perspect Biol. 2012; 4(12).

12. Kroemer G, Marino G and Levine B. Autophagy and the integrated stress response. Mol Cell. 2010; 40(2):280-293.

13. Jewell JL, Russell RC and Guan KL. Amino acid signalling upstream of mTOR. Nat Rev Mol Cell Biol. 2013; 14(3):133-139.

14. Sengupta S, Peterson TR and Sabatini DM. Regulation of the mTOR complex 1 pathway by nutrients, growth factors, and stress. Mol Cell. 2010; 40(2):310-322.

15. Tomomori-Sato C, Sato S, Conaway RC and Conaway JW. Immunoaffinity purification of protein complexes from Mammalian cells. Methods Mol Biol. 2013; 977:273-287.

16. Gera JF, Hazbun TR and Fields S. Array-based methods for identifying protein-protein and protein-nucleic acid interactions. Methods Enzymol. 2002; 350:499-512.

17. Martin J, Masri J, Bernath A, Nishimura RN and Gera J. Hsp70 associates with Rictor and is required for mTORC2 formation and activity. Biochem Biophys Res Commun. 2008; 372(4):578-583.

18. Masri J, Bernath A, Martin J, Jo OD, Vartanian R, Funk A and Gera J. mTORC2 activity is elevated in gliomas and promotes growth and cell motility via overexpression of rictor. Cancer Res. 2007; 67(24):11712-11720.

19. Shi Y, Sharma A, Wu H, Lichtenstein A and Gera J. Cyclin D1 and c-myc internal ribosome entry site (IRES)- dependent translation is regulated by AKT activity and enhanced by rapamycin through a p38 MAPK- and ERKdependent pathway. J Biol Chem. 2005; 280(12):1096410973.

20. Rice RL, Rusnak JM, Yokokawa F, Yokokawa S, Messner DJ, Boynton AL, Wipf P and Lazo JS. A targeted library of small-molecule, tyrosine, and dual-specificity phosphatase inhibitors derived from a rational core design and random side chain variation. Biochemistry. 1997; 36(50):1596515974.

21. Cloninger C, Bernath A, Bashir T, Holmes B, Artinian N, Ruegg T, Anderson L, Masri J, Lichtenstein A and Gera J. Inhibition of SAPK2/p38 enhances sensitivity to mTORC1 inhibition by blocking IRES-mediated translation initiation in glioblastoma. Molecular cancer therapeutics. 2011; 10(12):2244-2256.

22. Hsu PP, Kang SA, Rameseder J, Zhang Y, Ottina KA, Lim D, Peterson TR, Choi Y, Gray NS, Yaffe MB, Marto JA and Sabatini DM. The mTOR-regulated phosphoproteome reveals a mechanism of mTORC1-mediated inhibition of growth factor signaling. Science. 2011; 332(6035):13171322 .

23. Patterson KI, Brummer T, O'Brien PM and Daly RJ. Dualspecificity phosphatases: critical regulators with diverse cellular targets. Biochem J. 2009; 418(3):475-489.

24. Katagiri C, Masuda K, Urano T, Yamashita K, Araki Y, Kikuchi K and Shima H. Phosphorylation of Ser446 determines stability of MKP-7. J Biol Chem. 2005; 280(15):14716-14722.

25. Theodosiou A, Smith A, Gillieron C, Arkinstall S and Ashworth A. MKP5, a new member of the MAP kinase phosphatase family, which selectively dephosphorylates stress-activated kinases. Oncogene. 1999; 18(50):69816988.

26. Yang Q, Inoki K, Ikenoue T and Guan KL. Identification of Sin1 as an essential TORC2 component required for complex formation and kinase activity. Genes Dev. 2006; 20(20):2820-2832.

27. Zinzalla V, Stracka D, Oppliger W and Hall MN. Activation of mTORC2 by association with the ribosome. Cell. 2011; 144(5):757-768.

28. Chalhoub $\mathrm{N}$ and Baker SJ. PTEN and the PI3-kinase pathway in cancer. Annu Rev Pathol. 2009; 4:127-150.

29. Roux PP and Blenis J. ERK and p38 MAPK-activated protein kinases: a family of protein kinases with diverse biological functions. Microbiol Mol Biol Rev. 2004; 68(2):320-344.

30. Caunt CJ and Keyse SM. Dual-specificity MAP kinase phosphatases (MPKs): shaping the outcome of MAP kinase signaling. FEBS J. 2012; 280:489-504.

31. Bermudez O, Marchetti S, Pages G and Gimond C. Post-translational regulation of the ERK phosphatase DUSP6/MKP3 by the mTOR pathway. Oncogene. 2008; 27(26):3685-3691. 
32. Marchetti S, Gimond C, Chambard JC, Touboul T, Roux D, Pouyssegur J and Pages G. Extracellular signal-regulated kinases phosphorylate mitogen-activated protein kinase phosphatase 3/DUSP6 at serines 159 and 197, two sites critical for its proteasomal degradation. Mol Cell Biol. 2005; 25(2):854-864.

33. Dickinson RJ, Delavaine L, Cejudo-Marin R, Stewart G, Staples CJ, Didmon MP, Trinidad AG, Alonso A, Pulido R and Keyse SM. Phosphorylation of the kinase interaction motif in mitogen-activated protein (MAP) kinase phosphatase-4 mediates cross-talk between protein kinase A and MAP kinase signaling pathways. J Biol Chem. 2011; 286(44):38018-38026.

34. Cao W, Bao C, Padalko E and Lowenstein CJ. Acetylation of mitogen-activated protein kinase phosphatase-1 inhibits Toll-like receptor signaling. J Exp Med. 2008; 205(6):14911503.

35. Furukawa T, Sunamura M, Motoi F, Matsuno S and Horii A. Potential Tumor Suppressive Pathway Involving DUSP6/ MKP-3 in Pancreatic Cancer. The American Journal of Pathology. 2003; 162(6):1807-1815.

36. Schullerus D, Herbers J, Chudek J, Kanamaru H and Kovacs G. Loss of heterozygosity at chromosomes $8 p, 9 p$, and $14 \mathrm{q}$ is associated with stage and grade of non-papillary renal cell carcinomas. The Journal of Pathology. 1997; 183(2):151-155.

37. Emmert-Buck MR, Vocke CD, Pozzatti RO, Duray PH, Jennings SB, Florence CD, Zhuang Z, Bostwick DG, Liotta LA and Linehan WM. Allelic Loss on Chromosome 8p1221 in Microdissected Prostatic Intraepithelial Neoplasia. Cancer Research. 1995; 55(14):2959-2962.

38. Liao Q, Guo J, Kleeff J, Zimmermann A, Büchler MW, Korc $M$ and Friess H. Down-regulation of the dualspecificity phosphatase MKP-1 suppresses tumorigenicity of pancreatic cancer cells. Gastroenterology. 2003; 124(7):1830-1845.

39. Sato M, Vaughan MB, Girard L, Peyton M, Lee W, Shames DS, Ramirez RD, Sunaga N, Gazdar AF, Shay JW and Minna JD. Multiple Oncogenic Changes (K-RASV12, p53 Knockdown, Mutant EGFRs, p16 Bypass, Telomerase) Are Not Sufficient to Confer a Full Malignant Phenotype on Human Bronchial Epithelial Cells. Cancer Research. 2006; 66(4):2116-2128

40. Wang Z, Xu J, Zhou J-Y, Liu Y and Wu GS. MitogenActivated Protein Kinase Phosphatase-1 Is Required for Cisplatin Resistance. Cancer Research. 2006; 66(17):88708877.

41. Cui Y, Parra I, Zhang M, Hilsenbeck SG, Tsimelzon A, Furukawa T, Horii A, Zhang Z-Y, Nicholson RI and Fuqua SAW. Elevated Expression of Mitogen-Activated Protein Kinase Phosphatase 3 in Breast Tumors: A Mechanism of Tamoxifen Resistance. Cancer Research. 2006; 66(11):5950-5959.

42. Chan DW, Liu VWS, Tsao GSW, Yao K-M, Furukawa T, Chan KKL and Ngan HYS. Loss of MKP3 mediated by oxidative stress enhances tumorigenicity and chemoresistance of ovarian cancer cells. Carcinogenesis. 2008; 29(9):1742-1750. 